del Palacio del Rey, esté hoy extinta. En la actualidad, lo que existe en esa área hibridógena es una nutrida población de Onopordum nervosum, integrada por individuos acaules o con tallo muy pequeño, que recuerdan en gran medida a Onopordum acaulon L.

\section{BIBLIOGRAFÍA}

GONZÁLEZ SIERRA, G., C. PÉREZ MORALES, A. PENAS MERINO y S. RIVAS MARTÍNEZ -1992- Revisión taxonómica de las especies ibéricas del género Onopordum L. Candollea 47: 181-213.

LINDBERG, H. -1932- Itinera Mediterranea. Acta Soc. Sci. Fenn., nov. ser. B, 1 (2): 165.

MURBECK, SV. -1921- Celsia et Onopordon. Lunds Univ. Arsskrift, nov. ser., 18 (9): 15-18.

Dirección de los autores. Dpto. Biología Vegetal y Ecología. Universidad de Sevilla. Apdo. 1095 Sevilla. *Autor para correspondencia. stalavera@us.es

\title{
137. APORTACIONES Y CORRECCIONES AL GÉNERO OROBANCHE (OROBANCHACEAE) EN ANDALUCÍA
}

Antonio J. PUJADAS SALVÀ

Recibido el 2 de 0ctubre de 2008, aceptado para su publicación el 14 de octubre de 2008 Publicado «on line» en octubre de 2008

Contributions and corrections to the genus Orobanche (Orobanchaceae) in Andalusia.

Palabras clave: Cádiz, Orobanche, parásita, UICN.

Key words: Cadiz, IUCN, Orobanche, parasite.

Recientemente publicamos la actualización sobre el conocimiento del género Orobanche en Andalucía (Pujadas Salvà et al., 2007). En el tiempo transcurrido desde entonces hemos podido constatar la existencia de una novedad taxonómica, Orobanche densiflora var. tarifae A. Pujadas, var. nov., de interés para la Flora de Andalucía, además de algunos errores en las categorías UICN (2001) que se propusieron en dicho trabajo y que pasamos a corregir.

Orobanche densiflora Salzm. ex Reut.

Orobanche densiflora Salzm. ex Reut. in DC., Prodr. 11: 19 (1847) es una especie bien caracterizada por presentar tanto el tallo como las flores de un color blancoamarillento o a veces blanco, con 


\begin{tabular}{|c|c|c|}
\hline & O. densiflora & O. foetida \\
\hline Tallo, indumento & Densamente pubescente glandular & $\begin{array}{l}\text { Esparcidamente pubescente } \\
\text { glandular, raramente denso }\end{array}$ \\
\hline Tallo, pelos (mm) & $0,1-2$ & $0,1-1,3$ \\
\hline Inflorescencia $(\mathrm{cm})$ & $(5) 13-22 \times(2,5) 3-3,5$ & (5) $13-45 \times(2,3) 3-4,1$ \\
\hline $\begin{array}{l}\text { Infloresc encia, disposición } \\
\text { floral }\end{array}$ & Muy densa & $\begin{array}{l}\text { +/- densa en la parte superior, } \\
\text { laxa en la inferior, a veces toda } \\
\text { densa }\end{array}$ \\
\hline Cáliz (mm) & $9-12$ & $8-15$ \\
\hline Cáliz, segmentos & $\begin{array}{l}\text { Soldados abaxialmente, raramente } \\
\text { libres }\end{array}$ & $\begin{array}{l}\text { Soldados abaxialmente, } \\
\text { contiguos o libres }\end{array}$ \\
\hline Corola (mm) & (12)14-17 & (11)13-22 \\
\hline Corola, labio superior & $\begin{array}{l}\text { Escasamente emarginado, lóbulos } \\
\text { porrectos }\end{array}$ & Emarginado, lóbulos patentes \\
\hline Corola, labio inferior & $\begin{array}{l}\text { Lóbulo medio ligeramente mayor } \\
\text { que los laterales, porrecto o a veces } \\
\text { deflexo }\end{array}$ & $\begin{array}{l}\text { Lóbulo medio mucho mayor que } \\
\text { los laterales, deflexo }\end{array}$ \\
\hline Filamentos, indumento basal & $\begin{array}{l}\text { Densamente vilosos, } \\
\text { pelos } \leq 1 \mathrm{~mm}\end{array}$ & $\begin{array}{l}\text { Cortamente pubescentes, pelos } \leq \\
0,5 \mathrm{~mm} \text {, raramente glabros }\end{array}$ \\
\hline Anteras (mm) & $1,1-1,6$ & $1,5-2$ \\
\hline
\end{tabular}

Tabla 1. Principales diferencias morfológicas entre O. densiflora Salzm. ex Reut. y O. foetida Poir. sin tener en cuenta el color.

inflorescencia muy densa de ápice marcadamente comoso, los segmentos del cáliz soldados abaxialmente, raramente libres, la corola de (12)14-17 mm, glabrescente, y que en España se encuentra en las dunas costeras del la provicia de Cádiz, sobre Lotus creticus (Pujadas Salvà, 2002: 408).

Gracias a las referencias de Foley (2001: 54-56) y de Carlón et al. (2005: 36-40) pudimos localizar cerca del Faro Camarinal, Tarifa, Cádiz, una población de Orobanche densiflora formada por ejemplares típicos y además por abundantes individuos con una llamativa pigmentación rojo-púrpura en toda la planta excepto el estigma que es amarillo. No existen ejemplares de una coloración intermedia entre estas plantas y los $O$. densiflora típicos. Esas variantes de coloración rojo-púrpura han permanecido sin nombre (cf. Foley, 2001: 54-56; Carlón et al., 2005: 36-40) por lo que pasamos a denominarlas como: 
Orobanche densiflora var. tarifae A. Pujadas, var. nov.

Diagnosis: Cum typo optime congruens, sed caulis, folia, bracteae, calyx atque corolla omnia ruber-purpurea.

Holótipo: Cádiz, Tarifa, Playa del Cañuelo, c. Faro Camarinal, $36^{\circ} 5,383 \mathrm{~N} 5^{\circ}$ 48,326 W, 9 m, 10-V-2008, A. Pujadas, COA 45361 .

Derivatio nominis: "tarifae" de Tarifa, municipio de la provincia de Cádiz en cuya costa se encuentra el taxón.

Observaciones. En relación a esta nueva variedad, Carlón et al. (2005: 37) indican textualmente: "Esta última forma es con seguridad lo que ha dado pie a las respectivas citas locales que Pujadas \& al. (2003: 740, 742 ) hacen de $O$. foetida Poir. var. foetida y de $O$. foetida var. broteri (J.A. Guim.) Merino...". Este comentario se trata de una afirmación gratuita, hecha sin contrastar el material de referencia. En el Herbario COA no consta que lo hayan visto. Hemos revisado los pliegos testimonio y podemos afirmar que el correspondiente a $O$. foetida var. foetida "Punta Camarinal, Bolonia, arenal costero, pinares, 30STE4897, 19-VI-1996, A. Lora et al., COA 22085" está correctamente identificado, es un ejemplar robusto de $O$. foetida típico; la otra referencia de $O$. foetida var. broteri, "Zahara de los Atunes, junto al Faro Camarinal, sobre Scorpiurus muricatus, 30S0247175 / 3997612, 52 m, 10-V-2003, A. Pujadas, COA 31111", es un ejemplar de $O$. foetida correspondiente a la variante de porte menor, tallos menos robustos, corolas de tamaño menor, que denominamos como var. broteri (J.A. Guim.) Merino y que también está correctamente identificada; además, se trata de una planta localizada a 52 m.s.n.m., altura que no alcanzan las poblaciones de $O$. densiflora, por lo general no superan los 10 m.s.n.m. (Pujadas Salvà, 2002: 408), aunque, excepcionalmente encontramos algunas plantas que alcanzan los 33 m.s.n.m. [c. Faro Camarinal, bajada a la playa del Cañuelo (COA 45362)].

$O$. densiflora var. tarifae se podría llegar a confundir con $O$. foetida por presentar análogamente una coloración rojiza, aunque en el primer caso es de un color rojo-púrpura mientras que en el segundo es de un color rojo-sanguíneo. Para poder diferenciar claramente $O$. densiflora de $O$. foetida aportamos, en la Tabla 1, sus principales diferencias morfológicas sin tener en cuenta su color.

\section{Categorías UICN}

En Pujadas Salvà et al. (2007) propusimos las categorías UICN (2001) a nivel regional para cada taxón, con el rango de especie, subespecie o variedad, del género Orobanche presente en Andalucía. Sin embargo, a las plantas más comunes de Orobanche [O. nana (Reut.) Beck, O. mutelii F.W. Schultz subsp mutelii, O. latisquama (F.W. Schultz) Batt., O. gracilis Sm. subsp. gracilis, O. gracilis subsp. deludens (Beck) A. Pujadas, O. crenata Forssk., O. amethystea Thuill. subsp. amethystea], sobre las cuales no existe actualmente ningún grado de amenaza importante, se les atribuyó, por error, la categoría UICN: NT, casi amenazadas, cuando como máximo les corresponde la categoría UICN: LC, de preocupación menor.

AGRADECIMIENTOS. A José Santos Cabello Pérez por la versión latina de la diagnosis.

\section{BIBLIOGRAFÍA}

CARLÓN, L., G. GÓMEZ CASARES, M. LAÍNZ, G. MORENO MORAL, Ó. SÁNCHEZ PEDRAJA y G.M. SCHNEEWEISS -2005Más a propósito de algunas Orobanche L. y Phelipanche Pomel (Orobanchaceae) del oeste del Paleártico. Documentos Jard. Bot. Atlántico (Gijón) 3: 1-71. 
FOLEY, M.J.Y. -2001- Orobanche L., in: Paiva, Sales, Hedge, Aedo, Aldasoro, Castroviejo, Herrero \& Velayos (eds.), Flora iberica 14: 32-72. CSIC. Madrid.

PUJADAS SALVÀ, A.J. -2002- Orobanchaceae, in López-Sáez, Catalán \& Sáez (eds.). Plantas parásitas de la Península Ibérica e Islas Baleares, 345-451. Mundi-Prensa. Madrid.

PUJADAS SALVÀ, A.J., P. FRAGA I ARGUIMBAU, E. SÁNCHEZ GULLÓN \& N. MOLINA MAHEDERO -2003- Orobanche crinita and Orobanche foetida (Orobanchaceae) in the West Mediterranean area. Bocconea 16(2): 737-744.

PUJADAS SALVÀ, A.J., L. PLAZA ARREGUI, E. SÁNCHEZ GULLÓN, E. TRIANO
MUÑOZ, M. LÓPEZ MARTÍNEZ, C. BURGARELLA, D. RUBIALES OLMEDO, B. ROMÁN DEL CASTILLO, E. REYES SEPÚLVEDA y A. IVORRA JIMÉNEZ -2007El género Orobanche L. (Orobanchaceae) en Andalucía. Acta Bot. Malacitana 32: 91-126.

UICN -2001- Categorías de las Listas Rojas de la UICN. Versión 3.1. Comisión de la Supervivencias de las Especies de UICN. Gland y Cambridge.

Dirección del autor. Departamento de Ciencias y Recursos Agrícolas y Forestales. Edificio Celestino Mutis. Campus de Rabanales. Universidad de Córdoba. E-14071 Córdoba. Email: cr1pusaa@uco.es

\title{
138. SOBRE LA PRESENCIA EN MÁLAGA(ESPAÑA) DE TEUCRIUM OXYLEPIS FONT
} QUER

\author{
Baltasar CABEZUDO*, Andrés . V. PÉREZ LATORRE y Teresa NAVARRO
}

Recibido el 1 de septiembre de 2008, aceptadp para su publicación el 26 de septiembre de 2008. Publicado "on line" en octubre de 2008

Teucrium oxylepis in Malaga (Andalusia, Spain)

Palabras clave. Teucrium, Málaga, corología.

Key words. Teucrium, Málaga, chorology.

En el año 1987 recolectamos en el Parque Natural de la Sierra de las Nieves (Málaga) un ejemplar (MGC 22978) de una especie de Teucrium parecido a $T$. pseudoescorodonia Desf. pero que no pudimos identificar correctamente en base al estado fenológico tardío del material recolectado. Posteriormente (1997) y en una localidad cercana vimos otro ejemplar de la misma especie que aun tenía algunos cálices viejos y que en aquel momento creímos que correspondía, en base a sus caracteres 